

\title{
Les Grecs en Adriatique
}

Maria Paola Castiglioni ${ }^{1}$

\begin{abstract}
Résumé: Située au carrefour entre les péninsules balkanique et italienne et reliant les espaces méditerranéens aux régions de l'Europe centrale et septentrionale, la mer Adriatique a connu une importante fréquentation grecque à partir au moins du IXe s. av. J.-C. Attirés par les opportunités commerciales auxquelles les littoraux de cette mer permettaient d'avoir accès, les Grecs ne s'y sont cependant qu'exceptionnellement installés de façon permanente dans le cadre de l'apoikia. À travers une présentation synthétique, cette contribution vise à mettre en évidence comment la " mobilité " grecque dans cette mer s'est insérée dans des contextes historiques plus vastes et complexes (la " tyrannie » de Périandre, les projets hégémoniques de Denys de Syracuse) et à souligner l'impact des implantations coloniales ou emporiques grecques sur les populations locales.

Mots-clés: Contacts ; Mer Adriatique ; Emporia ; colonies phantômes.
\end{abstract}

Resumo: Situado no cruzamento entre as penínsulas Balcânica e Italiana, e conectando os espaços mediterrânicos das regiões da Europa central e setentrional, o mar Adriático experimentou uma importante presença grega a partir de pelo menos meados do século IX a.C. Atraídos pelas oportunidades comerciais às quais os litorais desse mar davam acesso, os gregos, no entanto, raramente se instalaram de forma permanente em estrutura de apoikia. Através de uma apresentação sintética, esta contribuição visa por em evidência como a "mobilidade" grega nesse mar está inserida nos contextos históricos mais vastos e complexos (a "tirania" de Periandro, os projetos hegemônicos de Dionísio de Siracusa) e destacar o impacto das implantações coloniais ou empóricas gregas nas populações locais.

Palavras-chave: Contatos; Mar Adriático; Emporia; Tirania; Colônias Fantasmas.

Abstract: Located at the crossroads between Balkan and Italian peninsulas and connecting Mediterranean
areas to central and northern Europe, the Adriatic Sea was widely frequented by Greeks from the Ninth
century BCE at least. Greeks were attracted to Adriatic coasts by trades, but they rarely established apoikiai.
This paper aims to highlight how Greek "mobility" in this sea has to be understood in the light of larger and
more complex historical contexts (the "tyranny" of Periander, the hegemonic projects of Dionysus of
Syracuse) and to define the effect of colonial settlements or Greek emporia on indigenous people.
Keywords: Contacts; Adriatic Sea; Emporia; Tyranny; Ghost Colonies.

En partant de la réflexion historiographique récemment renouvelée autour des mobilités méditerranéennes pendant la période antique et en raison de la complexité des échanges et de la multiplicité des présences qui ont occupé cette mer au cours de l'Antiquité, l'espace adriatique se présente comme un cas d'étude particulièrement stimulant ${ }^{2}$. En effet, la mobilité grecque s'est déclinée ici non seulement sous la forme de fondations coloniales, mais s'est aussi et surtout manifestée dans le cadre de trafics commerciaux

\footnotetext{
${ }^{1}$ Maître de conférences d'histoire grecque, Université de Grenoble Alpes, France.

2 Je renvoie à ce propos en particulier à la publication dirigée par CAPDETREY et ZURBACH, 2012 et à l'étude de D'ERCOLE, 2012.
} 
qui ont permis de disposer de points d'accès aux espaces continentaux, au nord comme au sud. Ce contexte « diasporique » a en outre facilité le contact entre groupes d’origines différentes installés, de façon temporaire ou permanente, sur les côtes adriatiques ou dans leur arrière-pays. L'Adriatique constitue ainsi également un terrain d'étude privilégié pour aborder les phénomènes des transferts culturels et pour réfléchir autour des questions des constructions identitaires et ethniques, qui s'expriment ici de façon tout à fait originale.

La mer Adriatique portait chez les Grecs deux noms distincts : Adrìas et Ionios ${ }^{3}$. Si le premier en indiquait à l'origine plutôt la partie septentrionale, qui semble se refermer sur les terres continentales de l'Europe centrale, et dérivait d'Adria (Tite-Live V 33), emporion fondé par les Etrusques au VI ${ }^{\mathrm{s}}$ s. av. J.-C., le deuxième toponyme, lonios kolpos ou lonios pontos, désignait en revanche l'ensemble des eaux adriatiques. L'expression Ionios poros définissait plus spécifiquement le Canal d'Otrante, le secteur le plus perméable aux influences méditerranéennes ${ }^{4}$.

La tradition reconnaît, aux origines de la dénomination d'lonios kolpos, le mythe des pérégrinations d'lo relaté par Eschyle : le poète tragique raconte que cette jeune fille d’Argos, transformée en génisse par Zeus, arriva par voie terrestre jusqu'à Dodone, en Épire, puis jusqu'à la mer qui, de " golfe de Rhéa ", changea son appellation en " golfe d’lo », en mémoire de son passage (Eschyle Prométhée enchaîné 836-841).

Une légende alternative transmise au II s. apr. J.-C. par Appien (Guerres civiles II 39), mais remontant sans doute à Théopompe, historien du IV' s. av. J.-C., voit en revanche, à l'origine du nom de la mer, un personnage éponyme: Ionios, fils de Dyrrhachios, petit-fils d'Epidamnos et arrière-petit-fils de Poséidon. Cette tradition est de toute évidence strictement liée à la naissance de la colonie corcyréenne d'Épidamne-Dyrrhachion, comme en témoignent les noms attribués à l'ascendance d'lonios, et met l'accent sur l'importance de cette fondation, la première véritable apoikia de l'Adriatique.

Il est inutile de souligner que ces mythes sont révélateurs de l'importance que cette mer avait aux yeux des Grecs, notamment ceux qui parcouraient ses eaux pour des raisons commerciales ou qui habitaient ses côtes, en particulier orientales, considérées comme plus propices à l'installation de ports, même si souvent les auteurs grecs nous livrent une géographie approximative, voire tout à fait fausse de ces littoraux et des populations qui y vivaient ${ }^{5}$. Le « golfe » adriatique a pourtant joué un rôle de carrefour commercial et culturel fondamental, depuis l'âge du bronze, époque des fréquentations mycéniennes, mettant en relation

\footnotetext{
${ }^{3}$ On ajoutera également à ces deux dénominations celle de " mer de Chronos ", enregistrée par Apollonios de Rhodes IV $327 ; 509 ; 548$ et faisant allusion à la partie septentrionale de la mer Adriatique. Sur cette appellation cf. DELAGE, 1930, p.210-211.

${ }^{4}$ M. Pallottino parlait à ce sujet de "mediterraneità" plus immédiate du Sud de l'Adriatique : PALLOTTINO, 1983, p.17. Je renvoie à la carte à la fin de l'article pour la localisation des lieux adriatiques cités (FIG. 1).

${ }^{5}$ Hérodote ne cite que trois fois l'Adriatique et ne se préoccupe pas d'en décrire les peuples. Thucydide n'en fait aucune mention. Cf. à ce propos : D’ERCOLE, 2005, p.172.
} 
les peuples indigènes (Illyriens, Messapiens, Dauniens, Picéniens, Ombriens, Étrusques et Vénètes) entre eux et avec les Égéens ${ }^{6}$.

\section{LES PREMIERS CONTACTS}

Hérodote (I 163) affirme que les premiers explorateurs grecs de la mer Adriatique (aussi bien que de la mer Tyrrhénienne et du « far West » ibérique) ont été les Phocéens, mais l'absence de traces matérielles de leur fréquentation rend difficile d'évaluer l'impact de leur apport ${ }^{7}$.

Selon une partie de la tradition littéraire, les premiers colonisateurs de la mer Adriatique auraient été en revanche les Eubéens: Plutarque fait allusion à une présence eubéenne à Corcyre, et le PseudoScymnos, au II s. av. J.-C., attribue la fondation d'Orikos, sur le littoral illyrien, à l'extrémité méridionale du golfe de Vlorë, à des Eubéens de retour de la guerre de Troie qui auraient échoué sur la côte adriatique à cause de vents défavorables (Plutarque Questions grecques 11 et Pseudo-Scymnos 441-443). La même légende liée aux nostoi est reprise par Pausanias, au sujet de la région de Thronion, ville vraisemblablement située à proximité de la colonie d'Apollonia et des monts Kérauniens, et fondée par un groupe de Locriens et d'Abantes (ainsi étaient désignés les Eubéens dans les poèmes homériques, Homère lliade II 536-545. Cf. BIFFI, 1985-86) lors de leur retour du conflit troyen (Pausanias V 22, 2-3). La présence eubéenne en Adriatique, affirmée par les textes anciens, mais non corroborée par les découvertes archéologiques, reste pourtant très problématique et seules des recherches plus poussées sur le terrain pourront fournir des réponses plus précises au sujet de ce qui semble être plus un contact précolonial qu'un établissement permanent $^{8}$.

La littérature, confortée cette fois par les découvertes archéologiques, livre en revanche des témoignages plus riches sur la présence corinthienne, au point que certains historiens ont pu parler de «Colonial Empire » à propos des fondations corinthiennes sur les rivages orientaux de la mer lonienne et du Sud de l'Adriatique, ou bien de " matrice corinzia » pour désigner l'empreinte politique et culturelle des Corinthiens dans ces régions 9 .

La première apoikia corinthienne, située en mer lonienne, mais à proximité du canal d'Otrante, aurait été fondée à Corcyre, sur la presqu'île située dans la partie méridionale de Corfou (site de Paléopolis), par une expédition guidée par Chersicratès, membre de la famille oligarchique des Bacchiades, qui détenait

\footnotetext{
${ }^{6}$ Pour ces époques, cf. en particulier LENZI, 2003.

${ }^{7}$ Sur le problème des Phocéens en Adriatique, cf. MOREL, 1975a, p.142-148; 1975b, p.853-896; 1982 , p.479-496 ; 1988 , p.31-44; 2001, p.53-77.

${ }^{8}$ Cf. à ce sujet le bilan, à propos d'Orikos en particulier, de BERETI et alii, 2011, p.410-430.

${ }^{9}$ Sur le débat, cf. QUANTIN, 2012, p.248 sq.
} 
alors le pouvoir à Corinthe, vers 734 av. J.-C., au moment où un autre Bacchiade, Archias, poursuivait sa navigation jusqu'à Syracuse, qu'il aurait fondée vers 733 av. J.-C. (Strabon VI 2, 4, 269) ${ }^{10}$.

Cette colonisation s'insère dans un réseau d'échanges bien établis à partir du début du VIII $\mathrm{s}$. av. J.-C., qui lient la ville de Corinthe à Ithaque par l'intermédiaire des sites d'Arta (Ambracie) et du village épirote de Vitsa (MORGAN, 1988). La découverte de céramique corinthienne de la même époque sur l'autre rive de l'Adriatique, à Otrante, atteste en outre de façon évidente l'intérêt des Corinthiens pour la route maritime qui conduisait vers l'Italie méridionale et la Sicile par le canal d'Otrante et dont Corfou constituait un relais important (D'ANDRIA, LOMBARDO, 1999).

Hérodote rapporte cependant que, depuis la colonisation de l'île, Corcyréens et Corinthiens étaient en désaccord, « bien qu'étant du même sang » (Hérodote III 49). Ce différend se serait concrétisé, aux dires de Thucydide, dans la première bataille navale connue par les Grecs, en 664, deux générations seulement après la fondation de la colonie (Thucydide I 38, 1). Ce combat naval, dont l'issue n'est pas révélée par les sources, aurait eu lieu au moment où l'organisation politique corinthienne connaissait un changement important, avec le passage du régime oligarchique mené par la famille des Bacchiades, à un gouvernement tyrannique guidé par Cypsélos, lui aussi Bacchiade par sa mère, mais en position marginale par rapport au groupe au pouvoir (Hérodote V 92$)^{11}$. Dans ce nouvel horizon, les orientations coloniales encouragées par les Bacchiades auraient été amplifiées par le tyran qui aurait confié à ses fils la fondation et le contrôle, en Acarnanie, le long de la route maritime qui conduisait à Corcyre et à la mer Adriatique, de Leucade, Anaktorion et Ambracie, au cours du VII ${ }^{\mathrm{e}}$ s. (Cf. QUANTIN, 2012, p.252-254, avec bibliographie).

\section{ÉPIDAMNE ET APOLLONIA}

La fondation des premières colonies de l'Adriatique, Épidamne-Dyrrhachion et Apollonia, dans une région occupée par les indigènes illyriens, se situe dans le même contexte.

La première fut fondée vers 627 av. J.-C., à l'initiative de Corcyre, sur un promontoire de la côte adriatique sud-orientale : selon la version enregistrée par Thucydide ( 24,2$)$, le contingent corcyréen fut conduit par un oikiste provenant de Corinthe, Phalios, fils d'Ératokleidès, corinthien de naissance et descendant des Héraclides. L'établissement adriatique d'Épidamne répond très certainement aux exigences de Corcyre, désireuse d'élargir et d'accroître son contrôle de la route maritime qui permettait de gagner l'Adriatique septentrionale, la plaine padane, les débouchés des routes alpines et d'entrer en contact avec les Étrusques installés en Émilie-Romagne. Bénéficiant de vents, de courants marins et d'abris favorables, la

\footnotetext{
${ }^{10}$ Sur les fondations presque contemporaines de Corcyre et de Syracuse, cf. INTRIERI, 2011, p.175-208.

${ }^{11}$ Plus en général, sur ces événements dans I'histoire de Corinthe : WILL, 1955 et SALMON, 1984.
} 
côte orientale de la mer Adriatique a été en effet largement préférée à la côte italienne, avec ses importuosa litora (Tite-Live $\times 2,4)^{12}$, pour les navigations en direction de la partie septentrionale du sinus Adriaticus (Strabon VII 5, 10). La colonie permettait en outre de disposer d'une base utile pour entrer en contact avec l'arrière-pays illyrien, comme en témoigne l'existence à Épidamne d'un magistrat chargé des transactions commerciales avec les indigènes illyriens, le polète (Plutarque Quaestiones graecae 297f).

L'apoikia d'Apollonia a été fondée un peu plus au sud et en correspondance de l'embouchure du fleuve Aôos (l'actuel Vjosa), à la même époque (Sur Apollonia, cf. DIMO, LENHARDT, QUANTIN, 2007), vers la fin du VII s. L'entreprise coloniale fut voulue sans doute par le fils et successeur de Cypsélos, Périandre (dont la tyrannie est traditionnellement datée entre 628 et 587), qui poursuivit ainsi la politique adriatique promue par les Bacchiades et renforcée par son père.

Les sources ne sont pourtant pas unanimes sur la composition de l'expédition coloniale : Thucydide, Pline I'Ancien, Dion Cassius et Stéphane de Byzance considèrent Apollonia comme une fondation corinthienne (Thucydide I 26, 2 ; Pline l'Ancien Histoire naturelle III 145 ; Dion Cassius XLI 45 et Stéphane de Byzance ss.vv. Apollonia et Gylakeia), alors que le Pseudo-Scymnos la présente comme une fondation mixte, corinthienne et corcyréenne à la fois (Pseudo-Scymnos 439-440). Pausanias semblerait enfin, dans un passage lacunaire et donc problématique, laisser aux seuls Corcyréens la responsabilité de l'initiative (Pausanias V 22, 4). Les contradictions des sources sont certainement le reflet de l'existence de traditions alternatives sur l'origine de cette cité créées au fil des siècles et des choix politiques de cette apoikia: dans leur interprétation, il faut tenir compte de la proximité géographique entre Apollonia et Corcyre, qui a engendré des relations étroites et a pu favoriser le développement et la diffusion d'une tradition de fondation philo-corcyréenne.

Stéphane de Byzance est le seul à conserver le nom de l'oikiste, le corinthien Gylax. Toujours selon ce lexicographe du $\mathrm{VI}^{\mathrm{e}}$ s. apr. J.-C., l'expédition était composée d'un contingent de deux cents colons corinthiens. Gylax baptisa la colonie du nom de Gylakeia, toponyme plus tard remplacé par celui d’Apollonia. Ce changement a dû probablement se faire après la chute du régime tyrannique des Cypsélides, vers 584/3 av. J.-C., lorsque la réaction au pouvoir tyrannique dans la métropole de Corinthe a pu avoir des répercussions politiques dans la colonie adriatique et a conduit ses habitants à rebaptiser la colonie du nom du dieu archégète par excellence, Apollon (Sur cette hypothèse, cf. ANTONELLI, 2000).

Gylakeia constituerait ainsi un cas tout à fait exceptionnel, à une date si ancienne, de colonie grecque prenant son nom de celui de son fondateur. Le privilège dont ce dernier profita découla probablement de son importante position au sein des fidèles du tyran Périandre : Gylax était peut-être l'un

\footnotetext{
${ }^{12}$ Sur les routes de navigation adriatique : ARNAUD, 2005, p. 194-206.
} 
de ses hommes de confiance, et aurait été chargé d'imposer à la nouvelle colonie adriatique une constitution modelée sur celle de Corinthe et de garantir la fidélité de la nouvelle fondation à sa métropole.

De la même façon et à la même époque, Périandre avait confié la fondation d'une autre colonie, Potidée, en Chalcidique, de l'autre côté de la péninsule balkanique, à son fils, Évagoras, et quelques années après, vers 594/3, le gouvernement de Corcyre à un autre de ses fils, Lycophron ou Nicolaos (Hérodote III 52 , 25 et Nicolas de Damas FGrHist 90 F 59, 1). Cela entraîna également la prise de contrôle de la colonie de Corcyre, Épidamne. Strabon précise en effet que, vers 588/87, Périandre envoya les réfugiés provenant de Dyspontion, en Élide, à Apollonia et à Épidamne, ce qui sous-entend un contrôle corinthien sur les deux colonies (Strabon VIII 3, 32, 357).

La position d'Apollonia est révélatrice : elle n'est pas installée directement sur la côte, mais dispose d'un port fluvial, vraisemblablement non loin de la mer, séparé de celle-ci par un espace lagunaire et marécageux formé par les méandres du fleuve Aôos, et domine une vaste plaine fertile, qui lui permet de se tourner vers une florissante activité agro-pastorale (CABANES, 2000). Cependant, la fondation d'Apollonia semble également répondre aux mêmes exigences commerciales que celles d'Épidamne. Elle pouvait en outre bénéficier de sa proximité avec les mines de bitume, localisées dans la région du nymphaion, aux frontières de la chora apolloniate ${ }^{13}$.

Les deux apoikiai adriatiques assuraient par ailleurs la maîtrise des routes reliant le littoral adriatique oriental à l'arrière-pays, notamment en suivant les cours du Genusus (Shkumbi) ou de l'Apsos (Seman), selon un tracé qui devient, à partir du $\|^{\mathrm{e}} \mathrm{s}$. av. J.-C., après la conquête romaine, celui de la via Egnatia, la principale route transbalkanique qui permettait de rejoindre, depuis Apollonia ou Épidamne, les régions nord-égéennes, macédoniennes et thraces, jusqu'aux Détroits ${ }^{14}$. La fondation corinthienne de Potidée, en Chalcidique, au débouché égéen de cette route continentale, semble du reste marquer une volonté de monopole de la part de Corinthe de cet itinéraire terrestre (Nicolas de Damas FGrHist 90 F 59, $1)^{15}$.

Les emplacements de ces deux colonies adriatiques auraient en outre favorisé un accès direct aux ressources minières de Damastion, situées à l'intérieur des terres, dans la région du lac Lychnitis (Ohrid) ${ }^{16}$.

Nombreux sont les témoignages de la prospérité de ces deux colonies dès leurs fondations : déjà en 572, parmi les prétendants de la fille du tyran de Sicyone, tous issus des meilleures familles du monde

\footnotetext{
${ }^{13}$ Sur les mines de bitume près d'Apollonia, cf. CABANES, 2004, p.124 et VREKAJ, 2011, p.199-207.

${ }^{14}$ Sur la via Egnatia, sur son parcours et sur les sources littéraires, épigraphiques et archéologiques qui en parlent, cf. FASOLO, 2005 et PALAZZO, 2010, p.273-290.

${ }^{15}$ L'expédition coloniale fut confiée à l'un des fils de Périandre, Évagoras.

${ }^{16}$ II n'est pas facile de situer précisément ce district minier pour lequel la source principale reste Strabon VII 7,8 et VIII 6,16 . Pour une synthèse sur le débat autour de son emplacement géographique, cf. CASTIGLIONI, 2010, p.115-116, avec les renvois bibliographiques.
} 
grec, figurait Amphimnestos d'Épidamne (Hérodote VI 127). Le sanctuaire panhellénique d'Olympie garde également les traces non seulement d'athlètes vainqueurs originaires des deux apoikiai (Kléosthénès d'Épidamne, vainqueur à la course de quadriges en 516, et Ménéptolémos d'Apollonia, vainqueur à la course du stade dans la catégorie des enfants entre 504 et 500 av. J.-C. ${ }^{17}$ ), mais aussi de monuments collectifs: vers le milieu du $\mathrm{VI}^{\mathrm{e}} \mathrm{s}$., les habitants d'Épidamne offrirent au Zeus olympien un trésor élevé sur la terrasse des trésors au-dessus du Métroôn, qui vint s'ajouter à ceux érigés par d'autres cités grecques (Pausanias VI 19). Un siècle plus tard, les Apolloniates dédièrent au même dieu un monument constitué d'une base en hémicycle sur laquelle étaient disposées, comme le révèle la description de Pausanias, les statues en bronze de Zeus, Héméra-Eos et Thétis au centre et, des deux côtés de la triade divine, sur les parties latérales, de cinq héros troyens et cinq héros achéens: Achille et Memnon aux extrémités de la base et, entre ceux-ci et le groupe central, Ulysse et Hélénos, Ménélas et Pâris, Diomède et Énée, Ajax et Déiphobos. Chaque héros achéen était disposé face à son ennemi troyen (Pausanias V 22, 2-4) ${ }^{18}$. L'épisode représenté sur l'exèdre était celui du duel d'Achille et Memnon pendant la guerre de Troie, raconté par Arctinos de Milet dans l'Éthiopide, un poème perdu du cycle troyen ${ }^{19}$. La dédicace du monument, enregistrée elle aussi par Pausanias et dont quelques fragments ont été retrouvés dans les années 1940 et 1950 lors des fouilles allemandes à Olympie, informait que l'offrande avait été financée avec la dîme du butin pris par les Apolloniates aux habitants de Thronion. Très probablement, cette petite ville, qui n'a pas encore été localisée avec certitude, se trouvait à la frontière méridionale de la chora apolloniate, et sa conquête permit à Apollonia d'élargir son contrôle territorial et de bénéficier de nouvelles terres fertiles à exploiter.

\section{CORCYRA MELAINA, LES ‘COLONIE FANTASMA’ ET LE DELTA DU PO}

Au nord d'Épidamne, la présence grecque paraît moins stable au moins à l'époque archaïque, si l'on excepte le cas de Korkyra Melaina, Corcyre la Noire, colonie établie sur l'île de Korčula par un contingent de Cnidiens aidés par des Corcyréens, dans le cadre d'une entente (philia) entre les deux cités. Plutarque affirme que cet accord fut la conséquence de la reconnaissance des Corcyréens envers les Cnidiens, car ces derniers avaient libéré trois cents jeunes Corcyréens appartenant aux meilleures familles de l'île et envoyés comme otages par Périandre au roi Alyatte de Lydie (Plutarque De Herodoti malignitate $22{ }^{20}$. Cela signifie que l'expédition coloniale fut possible seulement après la mort de Périandre et la perte successive du contrôle

\footnotetext{
${ }^{17}$ Pour Kléosthénès : Pausanias VI 10, 6. Cf. aussi MORETTI, 1957 : n 66. Pour Ménéptolémos : Pausanias VI 14, 13 et MORETTI, $1957: n^{\circ} 162$.

${ }^{18}$ Sur le monument des Apolloniates à Olympie : CABANES, 1993, CASTIGLIONI, 2004, ANTONETTI, 2010 et PICCININI, 2011.

19 II reste cependant le résumé de Proclos : cf. DEBIASI, 2004, p.124-178.

${ }^{20}$ Cf. aussi Pseudo Scymnos 426-430, Strabon VII 5, 5, Pline Histoire naturelle III 152. Sur la fondation de Corcyre la Noire, cf. MASTROCINQUE, 1988.
} 
corinthien sur Corcyre, donc au plus tôt au début du $\mathrm{VI}^{\mathrm{e}}$ s., au moment où les Cnidiens se dirigèrent vers la Sicile pour échapper à la pression exercée par Alyatte sur les cités grecques d’lonie (expédition de Pentathlos, cf. Diodore V 9, 4-5 et Pausanias X 16, 7). Le nom donné à la fondation témoignerait d'une volonté d’honorer les Corcyréens qui avaient soutenu les Cnidiens dans leur fondation coloniale dont l'emplacement n'est malheureusement pas déterminé du point de vue archéologique.

Le caractère éphémère ou la faiblesse des données disponibles sur d'autres établissements grecs adriatiques a conduit les historiens à forger la définition de 'colonie fantasma' (colonies fantômes, LOMBARDO, 2006, p.19-32) pour désigner des expériences coloniales temporaires, qui ont laissé des traces évanescentes et labiles dans la documentation littéraire et archéologique. Ce fut probablement le cas de la fondation citée par le psephisma de Lumbarda ${ }^{21}$, localisée sur l'île de Korčula, ou bien d'Elpie (Strabon XIV 2 , 10, et Stéphane de Byzance s.v. Elpia. Cf. VAN COMPERNOLLE, 1985, p.35-45), fondation rhodienne en Apulie, d’Anchialé, fondation parienne en Illyrie mentionnée seulement par Stéphane de Byzance (s.v. Anchialé), et d'Hérakleia et de son port, dont l'existence est signalée seulement par le Pseudo-Scylax (XXII) et dont la localisation est inconnue, ou encore du projet de colonisation athénien en Adriatique, sur lequel nous reviendrons.

Comme le souligne M. Lombardo, ces cas méritent d’être étudiés à la lumière de ce que D. Asheri désignait comme dynamiques de "décolonisation ", à savoir comme des établissements grecs qui, fondés pour répondre à des besoins d'ordre politique et militaire ou commercial, n'arrivent plus à survivre lorsque les objectifs à l'origine des projets coloniaux ne subsistent plus (Cf. ASHERI, 1996, p.73-115).

L'espace adriatique fut aussi le cadre d'expéditions inabouties: vers 530, selon Strabon, des Thessaliens auraient tenté de s'établir à Ravenne, sur la côte occidentale de l'Adriatique, mais ils rencontrèrent l'opposition des Étrusques, qui contrôlaient le delta du Pô (Strabon V 1, 7 c 214).

Les contacts entre Grecs et les populations de l'Adriatique centrale et septentrionale s'inscrivent toutefois dans des schémas autres que le modèle colonial classique, se déclinant plutôt sous la forme de relations emporiques nouées dans certains cas depuis l'âge du bronze. C'est les cas des Éginètes qui fréquentaient la région autour de l'emporion d'Adria, actif au moins depuis 580-570 et permettant d'entrer en contact avec les populations de la partie septentrionale de la mer Adriatique, le Caput Adriae, du delta du Pô au golfe de Venise (COLONNA, 1974, p.1-21).

\footnotetext{
${ }^{21}$ Cette inscription découverte à Lumbarda, dans I'île de Korčula, datée de la fin du IV $\mathrm{s}$. ou du début du III fait état d'une distribution de terres entre colons provenant d'Issa et répartis en trois tribus doriennes. Elle serait le témoignage de l'existence sur l'île, indépendamment de la colonie cnidienne de Corcyre la Noire, d'un établissement qui a probablement eu une fonction militaire de contrôle de la navigation côtière. Cf. LOMBARDO, 1993, p.161-188.
} 
Adria et Spina ${ }^{22}$, qui vit le jour en correspondance du bras méridional du Pô, ont livré une importante quantité d'importations grecques, comme en témoigne la quantité de vases attiques retrouvés dans les nécropoles de Valle Trebba et Valle Pega ${ }^{23}$. À ces objets s'accompagnait la présence d'individus d'origine grecque, notamment au cours $d u V^{e} s{ }^{24}$. La mention de l'édification d'un trésor des Spinètes à Delphes confirme la visibilité internationale de cette ville padane, aussi bien que sa puissance militaire, car l'offrande est consacrée en tant que dîme et est donc liée à une victoire militaire (peut-être en relation avec les activités de piraterie pour lesquelles les Étrusques étaient célèbres, cf. Strabon V 1, 7 c 214, mais aussi Denys d'Halicarnasse I 18, 3-5 et Pline III 115).

La richesse et la fréquentation de ces deux emporia étaient surtout liées à leur proximité avec les centres étrusques de la plaine padane (notamment Felsina, la future Bologne), et donc à la facilité d'approvisionnement en métaux, en ambre, qui arrivait dans le Haut-Adriatique depuis la Baltique, mais aussi en céréales de la plaine du Pô et en chevaux vénètes (Cf. SASSATELLI, 1993, p.211-213).

Il faut d'ailleurs souligner que la profusion de récits mythologiques localisés dans l'aire du delta du Pô et sur la côte vénète jusqu'au Timave (mythes de Phaeton et des Héliades, de Dédale, des Argonautes, des offrandes hyperboréennes, de Diomède) et la découverte de matériel égéen de l'âge du bronze et du fer fait supposer que cette partie de l'Adriatique était ouverte aux navigations grecques dès avant l'époque des fréquentations éginètes de la fin de l'époque archaïque (Cf. COLONNA, 1974, p.1-21; BRUNI, 1998, p.203220; BRACCESI, 1988, p.133-145).

\section{LA COLONISATION DU IVE S.}

À la question des colonies fantômes est étroitement lié le débat sur la présence syracusaine en Adriatique, d'Ancône à Lissos, opposant les défenseurs de l'idée d'un « empire " syracusain et les historiens qui, plus prudemment, tendent à nuancer la thèse d'une concrétisation effective des projets de Denys de Syracuse en Adriatique (Cf. WOODHEAD, 1970 et D'ANDRIA, 2002, p.117-137, part. 119) ${ }^{25}$.

Il est indéniable qu'une partie de la tradition littéraire reconnaît un rôle important à l'action du tyran syracusain en Adriatique au IV s. av. J.-C., et notamment entre 387 et 383 , dans le cadre d'un

\footnotetext{
${ }^{22}$ Sur Spina : Strabon V 1, 7 c 214, Pseudo-Scylax 17, Pline Histoire naturelle III 120, Trogue-Pompée XX 1, 11. Une synthèse très claire sur le site et sa tradition littéraire dans : TORELLI, 1993, p.53-69.

${ }^{23}$ En particulier, sur la nécropole de Valle Trebba, cf. BERTI, 1993, p.34-45.

${ }^{24}$ Sur le caractère multi-ethnique de ces deux emporia, cf. BOURDIN 2006, p.30 ss.

${ }^{25} \mathrm{En}$ réalité, le problème dépasse largement les frontières de l'Adriatique et demande une relecture générale de la "propagande dionysienne ", telle qu'elle nous est transmise par les sources, une confrontation de la politique adriatique du tyran avec sa politique tyrrhénienne, ses alliances illyrienne et épirote, l'évaluation de l'impact des projets, pas forcément concrétisés, de Denys sur le monde de la Grèce propre, et des réactions qu'ils ont déclenchées ici et à Athènes plus particulièrement. Sur ces questions, cf. BONACASA, BRACCESI, DE MIRO, 2002.
} 
programme de domination plus vaste. Après avoir apaisé le danger carthaginois (la troisième guerre contre Carthage se conclut favorablement pour Denys en 391 av. J.-C.), Denys élargit en effet ses visées expansionnistes au-delà de la Sicile. La première phase de son programme fut entreprise aux dépens des Étrusques, et mit à profit la descente des Celtes dans la plaine du Pô et en Italie centrale jusqu'à Rome, en 388 av. J.-C. Vraisemblablement, peu après cette date, Denys conclut une alliance avec les Gaulois et porta deux attaques successives contre l'étrusque Caere-Cerveteri et son emporion de Pyrgi : la première en 387/6, avec la collaboration de troupes mercenaires celtes provenant de l'lapygie, et la deuxième, entre 385/4 et 384/3, maritime, avec une flotte syracusaine (Diodore XIV 117, 7 et XV 14, 3-4). Ce furent là les premiers succès de son dessein d'hégémonie tyrrhénienne, réalisée seulement en partie (Diodore XVI 5,$4 ; 9,1 ; X X$ 78, 3. Cf. SINATRA, 1996, p.373-381 et SORDI, 1986, p.84-90).

À la même période, Denys entretenait des relations diplomatiques dans l'Adriatique : si l'on en croit Diodore (XV 13,1), celles-ci visaient à appuyer un vaste projet de contrôle balkanique dont l'opération culminante prévoyait le pillage du sanctuaire delphique, que les troupes du tyran auraient atteint en traversant les régions épirotes depuis le littoral adriatique. Le récit de Diodore dépend certes de sources peu objectives, toutefois une partie des notices sur la politique adriatique de Denys semble digne de foi: Diodore écrit en particulier que Denys, se servant de la médiation d'Alcétas le Molosse, fils de Tharyps, expulsé de son royaume par le parti philo-spartiate à la fin la guerre du Péloponnèse et en exil à Syracuse, s'allia avec le roi des Illyriens et lui envoya un contingent de deux mille soldats et cinq cent panoplies (Diodore XV 13, 2). Ce roi illyrien reste anonyme dans le récit de Diodore, mais il est fort probable qu'il s'agissait de Bardylis, roi des Dassarètes, tribu illyrienne située à la frontière entre Macédoine, Illyrie et Épire ${ }^{26}$. La collaboration militaire sicilienne conduisit à la restauration d'Alcétas sur le trône molosse et à la victoire finale de la coalition (Diodore XV 13, 3).

Un tel résultat orienta selon toute vraisemblance les équilibres adriatiques en faveur de Denys qui, profitant de l'entente avec les Illyriens du Sud, aurait décidé d'établir une base navale à Lissos (l'actuelle Lezhë) et d'intensifier son influence un peu plus au nord, dans le territoire où il avait déjà fondé la colonie d'Issa (dans l'actuelle île de Vis), en correspondance du plus large et du plus sûr des ports dalmates ${ }^{27}$.

En 385/4 av. J.-C., Denys aurait en outre prêté main forte aux Pariens pour la fondation de Pharos, l'actuelle Stari Grad, sur l'île de Hvar (Diodore XV 13, 3 et XV 14, 1-2. Sur Pharos, cf. KIRIGIN 2006). L'année suivante, il aurait renouvelé son soutien aux Pariens de Pharos en chargeant son gouverneur (éparchos) de leur porter secours lors d'une attaque de la part des Illyriens installés sur l'île et assistés par d'autres Illyriens

\footnotetext{
${ }^{26}$ Sur ce roi des Illyriens : Cicéron De officiis II 11 ; Diodore XVI 4, 4; Frontin Stratagèmes II 5, 19 ; Libanios Contre Sévère 52 ; Polybe XXXVIII 6, 4 et Théopompe FGrHist 115 F 28.

${ }^{27}$ Diodore XV 13, 4 (Lissos) et XV 14, 2, mais les manuscrits ne sont pas concordants, ce qui rend cette reconstitution incertaine et hypothétique. À ce sujet, cf. VIAL 1972, p.66-67. Le Pseudo-Scymnos 413-414, attribue à Issa une origine syracusaine.
} 
du continent. Il est en effet probable que la présence coloniale grecque ait été difficilement tolérée par les autochtones, probablement les Liburniens, dérangés surtout dans les activités de piraterie qu'ils menaient dans la mer Adriatique (Tite-Live X 2, 4 et Strabon VII 5, 10, c 317. Cf. FUSCAGNI, MARCACCINI, 2002, p.103113).

Selon une notice de Strabon (V 4, 2), la tyrannie de Denys aurait entraîné un groupe d'opposants au pouvoir du tyran à quitter la Sicile et à se réfugier à Ancône. Ce centre, qui dispose du seul bon port naturel du littoral occidental de l'Adriatique jusqu'au Gargano, était déjà occupé par un établissement indigène fréquenté par les Grecs. L'arrivée des exilés n'aurait fait qu'élargir cet emporion et en accroître la vocation commerciale (LUNI, 2004, p.28). Pline l'Ancien écrit que le site de Numana, peu loin au sud d'Ancône, aurait été lui aussi occupé par des "Sicules » (Pline Histoire Naturelle III 112), mais plus qu'à une installation syracusaine de l'époque de Denys, la notice plinienne serait une vague allusion aux migrations de la deuxième moitié du II millénaire (LUNI, 2004, p.56). Ici, comme à Ancône, la documentation archéologique montre que le site, établi à l'extrémité méridionale du promontoire du Conero, avait été habité par les indigènes dès l'âge du fer. Les importantes quantités de céramique attique retrouvées sur place confirment pour ce centre également une fonction d'escale maritime pour les navigants grecs qui parcouraient la route Zadar-Ancône.

Enfin, les fragments d'une stèle retrouvée au Pirée (IG II 1629$)$ renseignent sur un dessein athénien d'envoi d'une expédition coloniale en 325-324 en Adriatique (eis ton Adrian). Le décret (psephisma) définit quelques détails de ce projet, soumis par Céphisodon à l'ecclesia d'Athènes : l'objectif de l'expédition était de garantir un marché et une source d'approvisionnement en blé (emporia et sitopompia) aussi bien qu'une protection (phylakia) contre les Tyrrhenoi, les Étrusques, vraisemblablement les Étrusques padans. Si le nom de l'oikiste désigné est connu (Miltiade), la destination n'est malheureusement pas précisée. Le projet, de nature prioritairement commerciale (Athènes, touchée pendant ces années par une grave période de sécheresse, n'était plus en mesure de satisfaire ses besoins internes, même avec les importations provenant de la Mer Noire), resta très probablement inachevé. D'importants changements étaient en cours : Athènes, déjà affaiblie par la défaite de Chéronée, ne sut pas faire face à Alexandre et dut définitivement renoncer à ses prétentions d'assurer une politique de protection maritime, en Adriatique et ailleurs ${ }^{28}$.

Les conquêtes d'Alexandre et l'organisation du monde méditerranéen après sa mort ont entraîné également des conséquences dans l'espace adriatique, qui devint, à la fin du IV et au III s., le théâtre de l'intervention des Macédoniens et des luttes entre Illyriens et Épirotes ${ }^{29}$. Par la suite, l'appui militaire de Rome à Issa contre les pirates illyriens et leur reine Teuta, en 229-228 av. J.-C., avec la première guerre d'Illyrie, conduisit à la perte de l'indépendance politique des établissements grecs (Polybe II 2-12, Appien

\footnotetext{
${ }^{28}$ Sur cette inscription et son contexte historique : FERONE, 2004, p. 31-48 et ZAMBON, 2004, p. 147-172.

${ }^{29}$ Pour une présentation de l'histoire des colonies adriatiques pendant cette période, cf. CABANES, 2000, p. 67 ss.
} 
Illyrikè 7-8 et Dion Cassius fr. 49 = Zonaras VIII 19). Ceux-ci ont toutefois gardé une forte empreinte grecque et favorisé la diffusion de leur culture aux villes illyriennes à proximité, comme en témoignent les exemples d'Apollonia, qui a gardé son identité grecque, et de ses voisines illyriennes Byllis et Amantia, qui se sont dotées de structures urbanistiques typiquement grecques, telles le théâtre et le stade, et ont été enregistrées, vers 220 av. J.-C., dans la liste des théarodoques de Delphes : elles pouvaient donc participer aux concours pythiques, privilège accordé seulement aux Hellènes ${ }^{30}$, et furent donc de facto intégrées dans le monde grec.

Ce dernier exemple montre clairement qu'en dépit d'une présence coloniale moins diffuse par rapport à d'autres régions touchées par les "diasporas " grecques, l'influence grecque en Adriatique a largement dépassé le cadre strict des échanges économiques, en entraînant des processus d'acculturation dont l'intensité et la persistance furent tout à fait exceptionnels.

La compréhension des caractères et des raisons de la présence grecque en Adriatique est toutefois possible seulement à condition de l'analyser à la lumière des événements historiques qui ont marqué la vie des villes d'origine des colons et des marchands qui ont parcouru cette mer à partir du début de l'époque archaïque et jusqu'à la fin de l'Antiquité. Les étapes les plus marquantes ont été sans aucun doute celles de la politique corinthienne à l'époque des Cypsélides, de la tyrannie de Denys, des projets " thalassocratiques » d'Athènes à l'aube de la période hellénistique et finalement de la conquête romaine.

À l'origine de l'intérêt porté à cette mer par les Grecs, il y a indiscutablement sa situation géographique, qui en fait à la fois une passerelle et une frontière. Plus particulièrement, sa partie méridionale a joué un rôle de trait d'union essentiel entre l'arrière-pays balkanique (épirote et macédonien en particulier) et les régions situées au-delà du canal d'Otrante. Il suffira à ce propos de renvoyer aux synchronismes, soulignés par la tradition, entre les fondations de Corcyre et de Syracuse et au caractère éparpillé du " réseau » corinthien. Les sections centrale et septentrionale de l'Adriatique, qui permettaient l'accès à I'Italie padane et à l'Europe continentale, ont en revanche plutôt contribué à nourrir des échanges au vaste rayonnement.

Grâce au développement des recherches archéologiques, les traces de la présence des Grecs en Adriatique et de leur dialogue avec les autres groupes ethniques installés sur les côtes de cette mer se sont remarquablement enrichies. L'ouverture de nouveaux chantiers de fouilles, en particulier à partir des années 1990, a contribué à la définition progressive d'une "carte archéologique » de plus en plus dense de I'Adriatique antique, ce qui permet aujourd'hui d'intégrer cet espace dans une réflexion plus générale sur les mobilités grecques, le phénomène de la colonisation et, plus largement, les processus de contacts entre

\footnotetext{
30 PLASSART, 1921, p.1-85. Byllis figure en IV 37 (p.22) et Abantia-Amantia en IV 56 (p.23). Sur les rapports entre Grecs et indigènes en Illyrie Méridionale : CABANES, 2002, p.59 ss.
} 
Grecs et peuples non grecs. Cela laisse espérer le développement d'une interprétation historiographique plus nourrie de cet espace si complexe et si riche, trop souvent oublié dans les synthèses sur les mobilités grecques. Les conclusions négativistes du long article pionnier de R. L. Beaumont paru en 1936 (BEAUMONT, 1936: "The Adriatic coastlands were never fully hellenized»), sont donc aujourd'hui en bonne partie à nuancer ou plutôt à lire à la lumière des nouvelles approches épistémologiques et sur la base des résultats prometteurs de la recherche archéologique. 


\section{BIBLIOGRAPHIE}

ANTONELLI, Luca. Kerkyaikà, Ricerche su Corcira alto-arcaica tra lonio e Adriatico. Roma: L’Erma di Bretshneider, 2000.

ANTONETTI, Claudia. Tra storia ed epos: il donario degli Apolloniati a Olimpia (Paus. 5.22.2-4). IN: CINGANO, Ettore (a cura di), Tra Panellenismo e tradizioni locali. Generi poetici e storiografia. Alessandria: Edizioni dell'Orso, 2010, p.433-450.

ARNAUD, Pascal. Les routes de la navigation antique. Itinéraires en Méditerranée. Paris: Éditions Errance, 2005.

ASHERI, David. Colonizzazione e decolonizzazione. IN: SETTIS Salvatore (dir.), I Greci. Storia cultura arte società, I, Noi e i Greci. Torino: Einaudi, 1996, p.73-115.

BEAUMONT, R. L. Greek influence in the Adriatic sea before the fourth century B. C. Journal of Hellenic Studies, London: Council of the Society for the Promotion of Hellenic Studies, v. 56, p.159-204, 1936.

BERETI, Vasil, CONSAGRA, Gionata, DESCCEUDRES, Jean-Paul, SHPUZA, Saimir, ZINDEL, Christian. Orikos - la première colonie grecque en Adriatique? La première campagne de fouille albano-suisse. IN : LAMBOLEY, Jean-Luc, CASTIGLIONI, Maria Paola (éds.). L'Illyrie Méridionale et l'Épire dans I'Antiquité. V. Actes du V' Colloque international de Grenoble (8-11 octobre 2008). Grenoble : diff. De Boccard, 2011, p.410-430.

BERTI, Fede. Appunti per Valle Trebba, uno specimen della necropoli di Spina. IN: BERTI, Fede, GUZZO, Pier Giovanni (a cura di), Spina. Storia di una città tra Greci ed Etruschi. Ferrara: Comitato Ferrara arte, 1993. p.34-45.

BIFFI, Nicola. Elefenore e il nostos degli Abanti. Invigilata lucernis, Bari: Università di Bari, 7-8, p.77-98, 19851986.

BONACASA, Nicola, BRACCESI, Lorenzo, DE MIRO, Ernesto (a cura di). La Sicilia dei due Dionisî. Akragas 2. Atti della settimana di Agrigento, 24-29 febbraio1999. Roma: L'Erma di Bretshneider, 2002.

BOURDIN Stéphane. Fréquentation ou intégration: les présences allogènes dans les emporia étrusques et ligures (VIe-IVe siècles av. J.-C.). IN: CLÉMENT, François, TOLAN, John, WILGAUX Jérôme (éds.), Espaces d'échanges en Méditerranée. Antiquité et Moyen Âge. Rennes : Presses Universitaires de Rennes, 2006, p.19-39.

BRACCESI, Lorenzo. Indizi per una frequentazione micenea dell'Adriatico. IN: ACQUARO, Enrico, GODART, Louis, MAZZA, Federico (a cura di). Momenti precoloniali nel Mediterraneo antico: questioni di metodo, aree d'indagine, evidenze a confronto : atti del convegno internazionale (Roma, 14-16 marzo 1985). Roma: Consiglio nazionale delle ricerche, 1988, p.133-145. 
BRUNI, Stefano. Un problematico documento per la storia della frequentazione dell'area spinetica prima di Spina. Appunti sulle rotte adriatiche in età arcaica. IN: REBECCHI Fernando (a cura di). Spina e il delta Padano : riflessioni sul catalogo e sulla mostra ferrarese : atti del convegno internazionale di studi "Spina, due civiltà a confronto", Ferrara, Aula Magna dell'Università, 21 gennaio 1994. Roma : L'Erma di Bretschneider, 1998, p.203-220.

CABANES, Pierre. Apollonie et Épidamne-Dyrrachion : épigraphie et histoire. IN : CABANES, Pierre (éd.). L'Illyrie Méridionale et l'Épire dans l'Antiquité. II. Actes du Ile colloque international de ClermontFerrand (25-27 octobre 1990), Paris : diff. De Boccard, 1993, p.145-153.

CABANES, Pierre (dir.). Histoire de l'Adriatique. Paris : Éditions du Seuil, 2001.

CABANES, Pierre. La présence grecque sur la côte orientale de l'Adriatique en Illyrie du Sud. IN: CAMBI, Nenad, ČAČE, Slobodan, KIRIGIN, Branko (org.). Greek Influence along the East Adriatic Coast. Split : Književni Krug, 2002, p.51-63.

CABANES, Pierre. L'évergétisme à Apollonia d'Illyrie et à Dyrrachium, à l'époque romaine. IN : CÉBEILLACGERVASONI, Mireille, LAMOINE, Laurent, TRÉMENT, Frédéric (textes réunis par). Autocélébration des élites locales dans le monde romain. Contextes, textes, images (II s. av. J.-C.-III s. ap. J.-C.). Clermont-Ferrand : Presses universitaires Blaise-Pascal (Erga, Recherches sur l'antiquité, 7), 2004, p.121-128.

CAPDETREY Laurent, ZURBACH Julien (dir.). Mobilités grecques: mouvements, réseaux, contacts en Méditerranée de l'époque archaïque à l'époque hellénistique. Bordeaux: Éditions Ausonius, diffusion De Boccard, 2012.

CASTIGLIONI, Maria Paola. II monumento degli Apolloniati a Olimpia. Mélanges de l'École française de Rome - Antiquité, Roma : École française de Rome, 115-2, p.867-880, 2004.

CASTIGLIONI, Maria Paola. Cadmos-serpent en Illyrie. Itinéraire d'un héros civilisateur. Pisa : Pisa University Press, 2010.

COLONNA, Giovanni, I Greci di Adria. Rivista storica dell'Antichità. Bologna: Pàtron, 4, 1-2, p.1-21, 1974.

D'ANDRIA, Francesco, LOMBARDO, Mario (a cura di). I Greci in Terra d'Otranto. Galatina: Congedo, 1999.

D'ANDRIA, Francesco. L'Adriatico. I rapporti tra le due sponde: stato della questione, IN: BONACASA, Nicola, BRACCESI, Lorenzo, DE MIRO, Ernesto (a cura di). La Sicilia dei due Dionisî. Akragas 2. Atti della settimana di Agrigento, 24-29 febbraio1999. Roma: L’Erma di Bretshneider, 2002, p.117-137.

DEBIASI, Andrea. L'epica perduta. Eumelo, il Ciclo, l'Occidente (Hesperìa 20, Studi sulla grecità d'Occidente). Roma : L'Erma di Bretschneider, 2004.

DELAGE, Émile. La géographie dans les Argonautiques d'Apollonios de Rhodes. Paris : Féret et fils, 1930. 
D’ERCOLE, Maria Cecilia. Identités, mobilités et frontières dans la Méditerranée antique. L'Italie adriatique, $\mathrm{VIII}^{\mathrm{e}}-\mathrm{V}^{\mathrm{e}}$ siècle avant J.-C. Annales. Histoire, Sciences Sociales, Paris : Édition de l'École des hautes études en Sciences sociales, 2005/1, 60 année, p.165-181, 2005.

D’ERCOLE, Maria Cecilia. Histoires méditerranéennes. Paris : Éditions Errance, 2012.

DIMO, Vangjel, LENHARDT, Philippe, QUANTIN, François. Apollonia d'Illyrie. 1. Atlas archéologique et historique. Rome-Athènes : École Française d'Athènes, École Française de Rome, 2007.

FASOLO, Michele. La via Egnatia I. Da Apollonia e Dyrrachium ad Herakleia Lynkestidos. Roma: Istituto Grafico Editoriale Romano, 2005.

FERONE, Claudio. II IV secolo, Atene e I'Adriatico. IN: La pirateria nell'Adriatico antico, Hesperia 19, Roma: L'Erma di Bretshneider, p.31-48, 2004.

FUSCAGNI, Stefania, MARCACCINI, Carlo. Illiri, hostes communes omnium: I'immagine di una conquista. IN: MOSCATI CASTELNUOVO Luisa (a cura di). Identità e prassi storica nel Mediterraneo greco. Milano: Edizioni Et, 2002, p.103-113.

INTRIERI, Maria. Corcira tra Corinto e l'Occidente : rapporti e sincronismi di colonizzazione. IN: DE SENSI SESTITO, Giovanna, INTRIERI, Maria (org.). Sulla rotta per la Sicilia : L'Epiro, Corcira e l'Occidente. Pisa: Edizioni ETS, 2011, p.175-208.

KIRIGIN, Branko, Pharos the Parian settlement in Dalmatia: a study of a Greek colony in the Adriatic, BAR international series, Volume 1561. Oxford: Archeopress, 2006.

LENZI, Fiamma (org.). L'archeologia dell'Adriatico dalla Preistoria al Medioevo, Atti del convegno internazionale, Ravenna, 7-8-9 giugno 2001. Firenze: All'Insegna del Giglio, 2003.

LOMBARDO, Mario. Lo psephisma di Lumbarda: note critiche e questioni esegetiche. Hespería, 3, Roma: L’Erma di Bretshneider, p.161-188, 1993.

LOMBARDO, Mario. I Greci in Dalmazia. Presenze e fondazioni coloniali, IN: LENZI Fiamma (a cura di). Rimini e l'Adriatico nell'età delle guerre puniche. Atti del Convegno Internazionale di Studi. Rimini, Musei Comunali, 25-27 marzo 2004. Bologna: Ante quem, 2006, p.19-32.

LUNI, Mario. I porti di Ankon e Numana. IN: BRACCESI, Lorenzo, LUNI, Mario (a cura di). I Greci in Adriatico, 2. Hespería, 18. Roma: L'Erma di Bretshneider, 2004, p.11-56.

MASTROCINQUE, Attilio. Da Cnido a Corcira Melaina. Uno studio sulle fondazioni greche in Adriatico. Trento: Università degli Studi di Trento, 1988.

MOREL, Jean-Paul. Récentes recherches sur la colonisation phocéenne en Occident. Revue archéologique, Paris : Presses Universitaires de France, p.142-148, 1975a.

MOREL, Jean-Paul. L'expansion phocéenne en Occident. Dix années de recherches (1966-1975). Bulletin de Correspondance Hellénique, Athènes, Paris : École française d'Athènes, De Boccard, v. XCIX, p.853896, $1975 b$. 
MOREL, Jean-Paul. Les Phocéens d'Occident. Nouvelles données, nouvelles approches. La Parola del Passato, Napoli : Macchiaroli, v. XXXVII, p.479-496, 1982.

MOREL, Jean-Paul. Eubéens, Phocéens, même combat ? IN : BATS, Michel, D’AGOSTINO Bruno (org.). Euboica: I'Eubea e la presenza euboica in Calcidica e in Occidente. Atti del convegno internazionale di Napoli, 13-16 novembre 1996, Centre Jean Bérard, Istituto universitario orientale, Dipartimento del mondo classico e del Mediterraneo antico, Napoli : Centre Jean Bérard, diff. de Boccard, p.3144, 1998.

MOREL, Jean-Paul. Les Grecs entre l'Adriatique et la Tyrrhénienne. Anemos, Padova: Esedra, 2, p.53-77, 2001. MORETTI, Luigi. Olympionikai, i vincitori degli antichi agoni olimpici. Atti dell'Accademia dei Lincei - Memorie, Roma: Reale Accademia d'Italia, serie VIII, vol. 8, fasc. 2, p.55-198, 1957.

MORGAN, Catherine A. Corinth, the Corinthian Gulf and Western Greece during the Eighth Century BC. The Annual of British School at Athens, London: British School at Athens, 83, p.313-338, 1988.

PALAZZO, Silvia. "Ethne" e "poleis" lungo il primo tratto della " via Egnatia » : la prospettiva di una fonte. IN: ANTONETTI, Claudia (a cura di). Lo spazio ionico e la Grecia nord-occidentale. Territorio, società, istituzioni. Atti del Convegno Internazionale, Venezia, 7-9 gennaio 2010 (= Diabaseis 1). Pisa: Edizioni ETS, 2010, p.273-290.

PALLOTTINO, Massimo. Considerazioni sul problema della funzione storica dell'Adriatico nell'Antichità. IN: L'Adriatico tra Mediterraneo e penisola balcanica nell'Antichità (Lecce-Matera, 21-27 ottobre 1973), Taranto: Istituto per la storia e l'archeologia della Magna Grecia , 1983, p.11-21.

PICCININI, Jessica. Antitetagmenoi. Sul monumento degli Apolloniati a Olimpia. Saia. Annuario della Scuola archeologica italiana di Atene, Padova: Aldo Eusilio editore, vol. LXXXIX, serie III, 11, tomo 1, p.237250, 2011.

PLASSART, André. Inscriptions de Delphes, la liste des Théorodoques. Bulletin de correspondance hellénique. Athènes, Paris : École française d'Athènes, De Boccard, volume 45, p.1-85, 1921.

QUANTIN, François. Du même aux autres et de l'autre aux mêmes. Les Corinthiens sur les rives orientales de la mer Adriatique. Pallas, Toulouse : Service des publications de I'Université de Toulouse-Le Mirail, 89, p.247-274, 2012.

SALMON, John B. Wealthy Corinth. A History of the City to 338 BC. Oxford: Clarendon Press, 1984.

SASSATELLI, Giuseppe. La funzione economica e produttiva: merci, scambi, artigianato. IN: BERTI, Fede. GUZZO, Pier Giovanni (a cura di). Spina. Storia di una città tra Greci ed Etruschi. Ferrara: Comitato Ferrara arte, 1993, p.179-217.

SINATRA, Daniela. Dionisio e i Celti, Kokalos, Roma: Giorgio Bretschneider, XLII, p.373-381, 1996.

SORDI, Marta. Dionigi I, dinasta d'Europa. Contributi dell'Istituto di Storia Antica dell'Università del Sacro Cuore, Milano: Vita e Pensiero, XII, p.84-90, 1986. 
TORELLI, Mario. Spina e la sua storia. IN: BERTI, Fede, GUZZO, Pier Giovanni (a cura di). Spina. Storia di una città tra Greci ed Etruschi. Ferrara: Comitato Ferrara arte, 1993, p.53-70.

VAN COMPERNOLLE, Thierry. La colonisation rhodienne en Apulie : réalité historique ou légende ?, Mélanges de l'École française de Rome, Roma : École française de Rome, 97, I, p.35-45, 1985.

VIAL, Claude. Lissos et Issa, d'après deux passages de Diodore de Sicile. Cahiers d'Histoire. Lyon-GrenobleSaint-Étienne-Chambéry, Lyon : Centre historique du Centre-Est, tome XVII, p.66-67, 1972.

VREKAJ, Bashkim. Le Nymphée d'Apollonia d'Illyrie. IN : LAMBOLEY, Jean-Luc, CASTIGLIONI, Maria Paola (éds.). L'Illyrie Méridionale et l'Épire dans l'Antiquité. V. Actes du Ve Colloque international de Grenoble (8-11 octobre 2008). Grenoble : diff. De Boccard, 2011, p.199-207.

WILL, Édouard. Korinthiakà. Recherches sur l'histoire et la civilisation de Corinthe des origines aux guerres médiques, Paris : De Boccard, 1955.

WOODHEAD, Geoffrey. The 'Adriatic Empire' of Dionysius I of Syracuse. Klio, Berlin: Akad.-Verl München : Oldenbourg-Wiss.-Verl., Zeitschr.-Service, 52, p.503-512, 1970.

ZAMBON, Efrem. I provvedimenti contro i pirati in età ellenistica. IN: La pirateria nell'Adriatico antico, Hesperia 19, Roma: L’Erma di Bretschneider, 2004, p.145-172. 


\section{FIGURES}

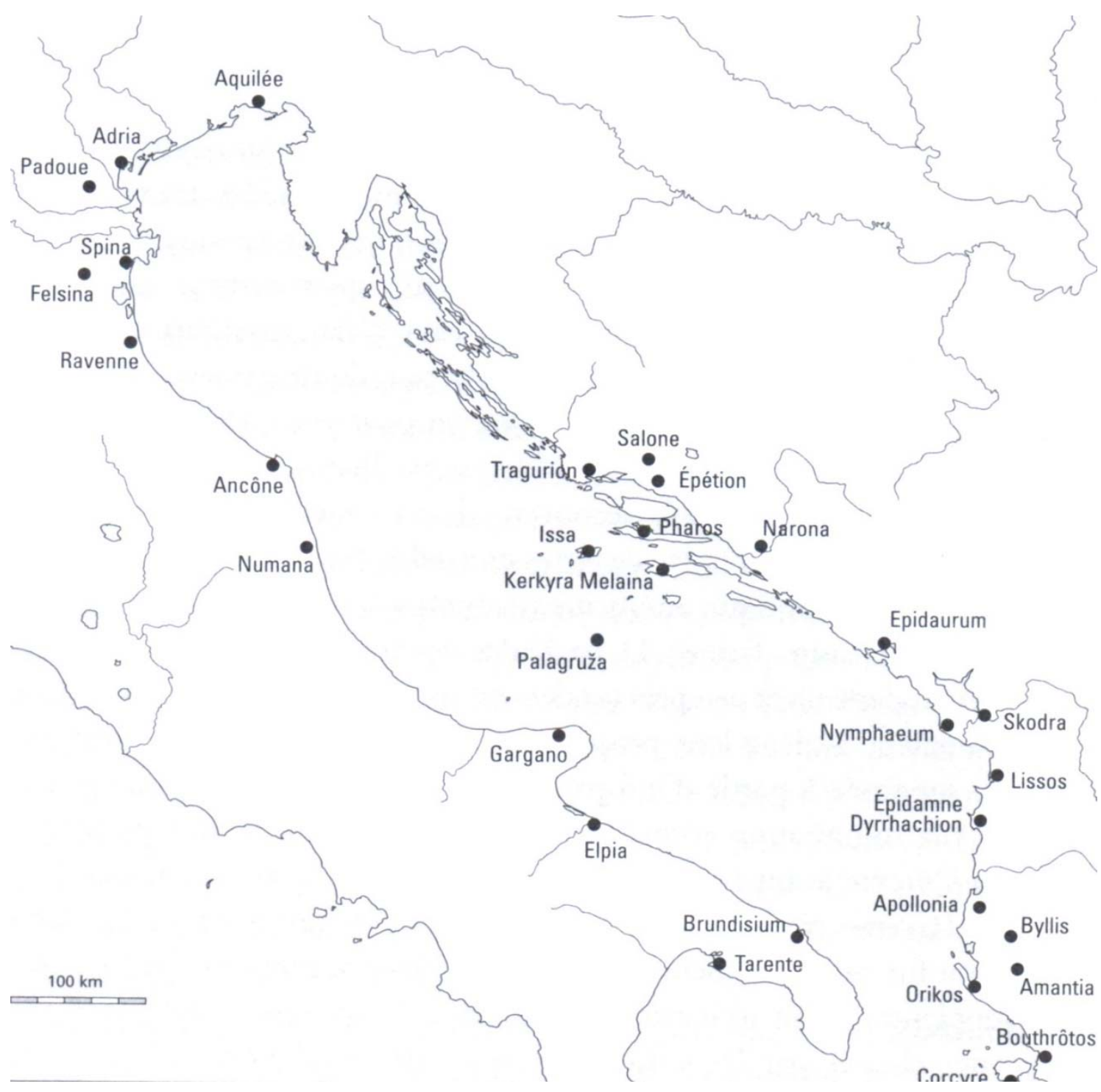

Figure. 1 : L'Adriatique grecque (Bouffier S., Les diasporas grecques. Du détroit de Gibraltar à I'Indus, Paris, Sedes, 2012). 